\title{
Perfuração do Septo Nasal: Etiologia e Diagnóstico
}

\author{
Perforation of Nasal Septum: Etiology and Diagnosis
}

\author{
Marco Aurélio Fornazieri *, Jemima Herrero Moreira**, Renata Pilan***, Richard Louis Voegels****. \\ * Otorrinolaringologista. \\ *** Fellowship em Cirurgia Endoscópica Endonasal e Cirurgia Plástica Facial. Otorrinolaringologista. \\ **** Fellowship em Cirurgia Endoscópica Endonasal da Divisão da Clínica Otorrinolaringológica do HC/FMUSP. Otorrinolaringologista. \\ ***** Professor Associado da Divisão de Clínica Otorrinolaringológica do Hospital das Clínicas da Faculdade de Medicina da Universidade de São Paulo. \\ Instituição: $\quad$ Faculdade de Medicina da Universidade de São Paulo. \\ São Paulo / SP - Brasil. \\ Endereço para correspondência: Disciplina de Otorrinolaringologia da Faculdade de Medicina da USP - Avenida Dr. Enéas de Carvalho Aguiar, 255 - 60 Andar - Sala 6167 \\ - São Paulo / SP - Brasil - CEP: 05403-000 - Telefax: (+55 11) 3088-0299 - E-mail : otorrino.ichc@hcnet.usp.br \\ Artigo recebido em 13 de Maio de 2009. Artigo aprovado em 10 de Agosto de 2009.
}

\section{RESUMO}

Introdução:

Objetivo:

Método:

Comentários Finais:

Palavras-chave:

\section{SUMMARY}

Introduction:

Objective:

Final Comments:

Keywords:
A perfuração do septo nasal é um achado ocasional da rinoscopia anterior e a maioria dos pacientes são assintomáticos. Contudo, são várias as etiologias possíveis dessa afecção, fazendo-se necessária uma investigação criteriosa.

Revisar na literatura as principais causas da perfuração septal e descrever os exames diagnósticos atualmente utilizados.

Revisão sistemática da literatura de periódicos indexados identificáveis até dezembro de 2008.

Entre as principais causas, encontram-se as traumáticas/iatrogênicas, o uso de drogas nasais, a exposição a gazes tóxicos, as doenças inflamatórias e infecciosas e as neoplasias. O diagnóstico se baseia na anamnese detalhada, com enfoque na ocupação e procedência do paciente, na observação das características mucosas da lesão, na biópsia e na coleta de exames complementares, como o ANCA, guiada pelas suspeitas principais.

septo nasal, diagnóstico, granuloma.

The nasal septum perforation is an occasional finding of rhinoscopy and most patients are asymptomatic. However, there are several possible etiologies of this condition, making necessary a thorough investigation. To review the literature the main causes of septal perforation and describe the diagnostic tests currently used. Method: A systematic literature review of journals indexed identifiable until December 2008. The main causes are the traumatic / iatrogenic nasal drug use, exposure to toxic gases, inflammatory and infectious diseases and neoplasms. The diagnosis is based on detailed medical history, focusing on occupation and origin of the patient, observation of the characteristics of mucosal injury on biopsy and collection of additional tests such as ANCA, guided by the main suspect.

nasal septum, diagnosis, granuloma. 


\section{INTRODUÇÃO}

As perfurações do septo nasal são achados ocasionais no exame otorrinolaringológico. A maioria dos pacientes são assintomáticos (1-3,4), sendo estimado que aproximadamente $2 / 3$ das pessoas acometidas não apresentem queixa nasal (1,4). A ausência de sintomas está relacionada diretamente ao tamanho da perfuração e a sua localização no septo nasal (5).

Muitas vezes, os pacientes procuram o otorrinolaringologista queixando-se de sibilos e formação de crostas nasais e descobrem possuir uma perfuração septal. Os sintomas mais discretos, como assobios discretos ocasionados pelo turbilhonamento de ar, estão relacionados às perfurações pequenas. As perfurações de tamanhos maiores estão relacionadas com o aparecimento de crostas, sangramentos, rinorreias, sensação de obstrução nasal, alterações do olfato, dor nasal, cefaleia e cacosmia $(6,7,8,9)$. Se a umidade nasal está preservada, a perfuração septal geralmente é assintomática. Quanto mais anterior, mais frequente a presença de sintomas $(3,10,11)$.

São várias as etiologias da perfuração septal (PS). A causa mais comum é a de origem iatrogênica por laceração do mucopericôndrio bilateralmente durante uma septoplastia ou por formação de hematoma pós-cirúrgico comprometendo a nutrição da cartilagem quadrangular do septo. Contam também entre suas causas as diversas doenças granulomatosas (leishmaniose, hanseníase, granulomatose de Wegener, rinoscleroma, sífilis, entre outras), traumas, por exemplo, cauterização química para epistaxes, uso de entorpecentes, principalmente a cocaína, potente vasoconstritor, além de drogas utilizadas para tratamento de patologias nasais como corticosteroides e vasoconstritores nasais.

Neste artigo, salientamos as principais causas de perfuração do septo nasal e revisamos os métodos diagnósticos atualmente utilizados. É importante ressaltar que os artigos recentes abordam principalmente o manejo cirúrgico do fechamento das perfurações septais, sendo menos discutido na literatura uma abordagem mais ampla sobre a etiologia e o diagnóstico.

\section{Etiologia}

As perfurações acontecem por injúria à cartilagem septal causada pela perda da integridade do mucopericôndrio que a reveste, com interrupção do aporte sanguíneo e consequente necrose. Essas injúrias podem ser iatrogênicas, traumáticas, inflamatórias, neoplásicas, infecciosas ou pela inalação de irritantes (5). A tabela a seguir mostra as principais causas de perfuração septal (Tabela 1).
Tabela I. Etiologia da perfuração septal.

\begin{tabular}{|c|c|}
\hline Traumática/latrogênicas & $\begin{array}{l}\text { Cirurgia Septal } \\
\text { Cauterização Química } \\
\text { Tamponamento Nasal } \\
\text { Sonda Nasogástrica } \\
\text { Intubação Nasal Prolongada } \\
\text { Fratura Nasal } \\
\text { Corpo Estranho } \\
\text { Manipulação Digital }\end{array}$ \\
\hline Uso de Drogas Nasais & $\begin{array}{l}\text { Descongestionante Nasal } \\
\text { Corticoide Nasal } \\
\text { Cocaína }\end{array}$ \\
\hline Exposição Ocupacional & $\begin{array}{l}\text { Vapores de Crômio eÁcido Sulfúrico } \\
\text { Limalha de Ferro } \\
\text { Pó de Vidro }\end{array}$ \\
\hline Inflamatória & $\begin{array}{l}\text { Granulomatose de Wegener } \\
\text { Sarcoidose } \\
\text { Lúpus Eritematoso Sistêmico } \\
\text { Artrite Reumatoide } \\
\text { Doença de Crohn } \\
\text { Dermatomiosite } \\
\text { Sarcoidose }\end{array}$ \\
\hline Infecciosa & $\begin{array}{l}\text { S'́flilis } \\
\text { HIV } \\
\text { Infecções Fúngicas } \\
\text { Hanseníase } \\
\text { Tuberculose } \\
\text { Leishmaniose } \\
\text { Rinoscleroma }\end{array}$ \\
\hline Neoplasias & $\begin{array}{l}\text { Carcinomas } \\
\text { Linfomas }\end{array}$ \\
\hline Idiopática/Indefinida & ? \\
\hline
\end{tabular}

As causas iatrogênicas e traumáticas são as mais prevalentes e ocorrem após cirurgias de septoplastia, cauterizações septais, radioterapia e tamponamentos nasais prolongados $(1,3,5,10,12,13)$. O uso prolongado de sonda nasogástrica também é descrito como possível causador de perfurações septais (5). A presença da perfuração no pós-operatório decorre de lacerações contrapostas no mucopericôndrio, injúria na cauterização e perda do suprimento sanguíneo pela elevação do flap mucopericondral (14). Dos pacientes submetidos a cirurgia nasal, os homens são os mais acometidos. Explica-se essa prevalência maior no sexo masculino pelo maior número de septoplastias entre os homens $(13,15)$.

As perfurações septais pós-septoplastia são encontradas entre $1 \%$ e $8 \%$ dos pacientes em investigação. Alguns autores citam que o número de perfurações aumenta quando se adota a técnica de Killian, caracterizada pela 
ressecção submucosa sem abordagem da porção caudal do septo. Já na técnica de Cottle, em que se aborda a porção caudal do septo, as complicações mais frequentes são outras: deslocamentos e instabilidade pós-operatórios (1619).

As causas traumáticas mais comuns são as fraturas nasais, rinolitos, corpos estranhos, hematomas septais e manipulação digital repetidas $(1,5,7)$.

O uso crônico de inalantes irritativos como descongestionantes nasais e cocaína podem levar a necrose da cartilagem pelo efeito vasoconstritor local, com consequente isquemia, e também pelo próprio componente cáustico presente em algumas de suas composições (10). O uso de corticoesteroides nasais por longo prazo também é descrito como agente causador de perfurações $(5,20)$, particularmente no sexo feminino (21). E a associação de corticosteroide e descongestionante nasal parece aumentar a ocorrência de perfurações (22).

Além dos agentes irritativos já citados, são descritos na literatura inúmeras substâncias relacionadas a PS: poeiras químicas e industriais (vapores de cromo, cobre, sal, ácido sulfúrico e clorídrico, pó de cimento, limalha de ferro, piche, pó de vidro, carbonato de sódio, óxido de cálcio, cianeto de cálcio, arsenicais, mercuriais, fósforo e benzeno) e aerossóis usados na agricultura.

As rinossinusites bacterianas e fúngicas são causas infecciosas dessa afecção. Contam também dentro desse grupo a sífilis, HIV, tuberculose, rinoscleroma, rinoesporidiose, paracoccidioidomicose e abscessos septais. Ainda entre as causas infecciosas estão a leishmaniose cutânea e a hanseníase, que ainda apresentam alta prevalência no Brasil, com um aumento do número de casos notificados em todas as regiões $(7,23,24)$.

A granulomatose de Wegener e a sarcoidose são as doenças inflamatórias mais comumente associadas a perfuração septal (25). Outras alterações vasculares e do colágeno, caso do lúpus eritematoso sistêmico, ocasionam também degeneração do septo nasal.

As neoplasias nunca devem ser esquecidas no diagnóstico diferencial das perfurações septais. As mais comumente associadas são o carcinoma espinocelular, crioglobulinemia e linfomas de células T $(5,26)$.

\section{Diagnóstico}

A presença de perfuração septal é facilmente diagnosticada pelo otorrinolaringologista durante sua anamnese e exame físico e sua etiologia dificilmente é

\section{Tabela 2. Abordagem diagnóstica.}

Anamnese

Incluir procedência, profissão, uso

de drogas nasais, cirurgias e

tratamentos prévios, sintomas constitucionais (emagrecimento, febre, tosse, artralgia, hematúria, áreas de pele sem sensibilidade) Inquérito geral sobre possíveis etiologias

Exame Físico / Endoscopia nasal

Observartamanho, dimensão, localização da PSAvaliar bordos da PS e características da mucosa adjacente

Biopsiarárea suspeita Geralmente é realizada no bordo posterior da PS e, se possível, incluir na amostra mucosa sadia e suspeita.

Avaliar necessidade de Descritas no quadro 3 exames complementares

definida. Uma maior elucidação diagnóstica é decorrente do seguimento dos seguintes passos: questionamento sobre sintomas nasais, história de uso de medicamentos prévios e hábitos sociais, rinoscopia anterior, nasofibroscopia, averiguação da perfuração septal quanto a aspecto, tamanho e localização.

Primeiramente, o diagnóstico depende da obtenção de uma história completa, ressaltando as cirurgias, tratamentos nasais prévios e uso prévio de inalantes irritativos, como a cocaína (10) Tabela 2.

Quando sintomático, o paciente apresenta-se com queixas típicas de respiração ruidosa, formação de crostas, obstrução nasal, rinorreia, ressecamento nasal, dor nasal e epistaxes. Essas queixas são explicadas principalmente pelo fluxo aéreo nasal turbulento. A perda do fluxo laminar nasal leva a formação de crostas nas margens da perfuração que, por sua vez, resulta nos demais sinais e sintomas citados. O nariz seco leva a formação de crostas, responsáveis pela epistaxe subsequente, mau odor e obstrução nasal. Os sibilos são consequência direta do fluxo aéreo pela perfuração (10).

No exame físico, é importante avaliar a presença de manchas esbranquiçadas na pele com perda de sensibilidade e espessamento de nervos, que indicam diagnóstico de hanseníase.

No exame otorrinolaringológico, na maioria das vezes, durante a rinoscopia anterior pode ser visualizada a perfuração septal. 
As perfurações septais podem ser classificadas quanto ao tamanho em pequena (até1 cm), média (1 a $2 \mathrm{~cm}$ ) e grande (maior que $2 \mathrm{~cm}$ ). A aferição do tamanho é importante não só pela questão legal, mas principalmente para a escolha da melhor opção terapêutica (10). Essa medição pode ser feita de várias formas, todas de realização muito simples. A primeira que a literatura aborda é a aferição com régua pelo lado oposto ao que se está realizando a rinoscopia. Outra técnica consiste em colocação de fio cirúrgico até margem posterior da perfuração e, com uma pinça hemostática, marcar o ponto da margem anterior, realizando depois medida com régua. Finalmente, pode ser utilizada pasta de bário nas bordas da perfuração e, através de uma radiografia em perfil, delimitar o tamanho da perfuração.

Quanto ao tamanho da perfuração, no levantamento de Pedroza avaliando 68 pacientes, foi observado que $12 \%$ apresentavam perfurações pequenas, $57 \%$ médias e $31 \%$ lesões grandes (13).

Após fácil diagnóstico estrutural, maior problema se encontra após, quando se procura as causas da perfuração.

Uma rinoscopia demonstrando mucosa hiperemiada e secreção purulenta abundante supõe uma causa infecciosa para a perfuração, bacteriana ou fúngica. A infecção fúngica acomete principalmente indivíduos imunocomprometidos. Confirma-se a infecção fúngica através de uma história compatível e biópsia do tecido infectado. Convém que o tecido seja enviado fresco ao laboratório e sem contato prévio com gazes, cuidados que facilitam a caracterização da infecção fúngica (10).

Os exames complementares na investigação da etiologia da perfuração septal estão na Tabela 3.

Como visto, devido às várias possíveis etiologias da perfuração septal a avaliação laboratorial pode ser muito ampla. Cabe ao otorrinolaringologista, através da história clínica e exame físico, discernir quais são os exames mais adequados para cada caso.

A biópsia é de extrema importância no diagnóstico etiológico da perfuração septal e deve ser realizada na investigação inicial. Além de excluir ou confirmar a presença de neoplasias como o carcinoma epidermoide (27), faz o diagnóstico diferencial de várias doenças. No caso das doenças inflamatórias, por exemplo, a presença de vasculite complementa o diagnóstico de granulomatose de Wegener, enquanto o achado de granulomas nãocaseosos indicaria um quadro de sarcoidose (10). Se a suspeita de neoplasia persistir, deve ser repetida biópsia até elucidação diagnóstica.
Tabela 3. Exames complementares.

\begin{tabular}{ll}
\hline Biópsia & Coloração \\
& Cultura \\
& Imunoistoquímica \\
& Hemograma \\
Séricos & VHS \\
& FAN \\
& ANCA \\
& FR \\
& Cálcio \\
& Dosagem de ECA \\
& Leishmaniose \\
& Paracoccidioidomicose \\
Sorologias & Histoplasmose \\
& Aspergilose \\
& VDRL / FTAabs \\
& Montenegro \\
Reações Intradérmicas & PPD \\
& \\
Urinal & \\
RXTórax & \\
Escarro - Pesquisa de BAAR & 3 amostras \\
Swab Nasal & Pesquisa de BAAR \\
\hline
\end{tabular}

\section{COMENTÁRIOS FINAIS}

A etiologia da perfuração septal deve ser buscada em todos pacientes. Uma anamnese completa seguida de rinoscopia anterior, nasofibrocospia e exames complementares adequados para cada caso, com destaque para a biópsia da lesão, constituem os principais meios a elucidação diagnóstica e subsequente correto manejo terapêutico.

\section{REFERÊNCIAS BIBLIOGRÁFICAS}

1. Brain DJ. Septo-rhinoplasty: The closure of septal perforations. J Laryngol Otol. 1980, 94(5):495-505.

2. Olieveira RCB, Mateus AR, Augusto AG. Perfuração septal. Escolha da técnica cirúrgica. Acta ORL. 2006, 24(3):134-138.

3. Tasca I, Compadretti, GC. Closure of nasal septal perforation via endonasal approach. Otolaryngology-Head and Neck Surgery. 2006, 135(6):922-927.

4. Eng SP, Nilssen EL, Ranta M, et al. Surgical Management of septal perforation: an alternative to closure of perforation. J Laryngol Otol. 2001, 115(3):194-197.

5. Metzinger SE, Guerra AB. Diagnosing and treating nasal septal perforations. Aesthetic Surg J. 2005, 25(5):524-9. 
6. Vicenti AB, Lourenço EA, Morgado PF. Cirurgia da perfuração do septo nasal. Em: Campos CAH, Costa HOO, editors. Tratado de otorrinolaringologia. $1^{a}$. ed. São Paulo: Roca; 2003, 5:248-59.

7. RE M, Paolucci L, Romeu R, Mallardi V. Surgical treatment of nasal septal perforations: our experience. Acta Otorhinolaryngol Ital. 2006, 26(2):102-109.

8. Shikowitz, MJ. Vascularized Mucoperiosteal Pull Through Flap for Closure of Large Septal Perforation: A new technique. Laryngoscope. 2007, 117(4):750-755.

9. Kridel RWH. Septal perforation repair. Otolaryngol Clin North Am. 1999, 32(4):695-724.

10. Coleman Jr JR, Strong EB. Management of nasal septal perforation. Current Opinion in Otolaryngology \& Head and Neck Surgery. 2000, 8(1):58-62.

11. Foda HMT, Magdy EA. Combining Rhinoplasty with Septal Perforation repair. Facial Plastic Surgery. 2006, 22(4):281288.

12. Belmont JR. An aproach to large nasosseptal perforations and attendant deformity. Arch Otolaryngol. 1985, 111(7):450-455.

13. Pedroza F, Patrocínio LG, Arevaldo . A review of 25-year experience of nasal perforation repair. Arch Facial Plast Surg. 2007, 9:12-18.

14. Bent JP, Wood BP. Complications resulting from treatment of severe posterior epistaxis. J Laryngol Otol. 1999, 103:66-75.

15. Schulz-Coulon, H-J. Experiences with the bridge flap technique for the repair of large nasal septal perforations. Rhinology. 1994, 32(1):25-33.

16. Gubisch W. Extracorporeal septoplasty for the markdley deviated septum. Arch Facial Plast Surg. 2005, 7(4):218-226.

17. Baterman ND, Woolford TJ. Informed consentt for septal surgery: the evidence-base. J Laryngol Otol. 2003, 117:186189.

18. Bewarder F, Pirsig W. Long-term results of submucous septal ressection. Laryngol Rhinol Otol (Stuttg). 1978, 57(10):922-931.

19. Rettinger G, Kirsche H. Complications in septoplasty. Facial Plast Surgery. 2006, 22(4):289-297.

20. Kridel RWH. Considerations in the etiology, treatment, and repair of septal perforations. Facial Plast Surg Clin N Am. 2004, 12(4):435-450.

21. Cervin A, Andersson M. Intranasal steroids and septum perforation - an overlooked complication? A description of the course of events and a discussion of the causes. Rhinology. 1998, 36(3):128-32.

22. Døsen LK, Haye R. Nasal septal perforation 1981-2005. Changes in etiology, gender and size. BMC Ear, Nose and Throat Disorders. 2007, 7:1.

23.Martins ACC, Castro JC, Moreira JS. Estudo retrospectivo de dez anos em endoscopia das cavidades nasais de pacientes com hanseníase. Rev Bras Otorrinolaringol. 2005, 71(5):609-16.

24. Falqueto A, Sessa PA. Leishmaniose Tegumentar Americana. Em: Veronesi R, Focaccia R, editores. Tratado de Infectologia. $3^{\underline{a}}$ ed. Rio de Janeiro: Atheneu; 2005, p.154559.

25. Baum ED, Boudousquie AC, Li S, Mirza N. Sarcoidosis with nasal obstruction and septal perforation. ENT J. 1998, 77(11):896-902.

26. Knudsen SJ, Bailey BJ. Midline Nasal Masses. Em: Head \& Neck Surgery - Otolaryngology. $3^{\text {rd }}$ edition. Lippincott Willians \& Wilkins Publishers; 2003. chapter 27.

27. Goulart IMB, Patrocínio LG, Nishioka SA, PatrocínioJA, Ferreira MS, Fleury RN. Concurrent Leprosy and leishmaniasis with mucosal involvment. Lepr Rev. 2002, 73(3):283-284. 